\title{
LA FORMACIÓN PROFESIONAL CRISTIANA Y LA VOCACIÓN DE SERVICIO DE LOS DOCENTES DE EDUCACIÓN RELIGIOSA DE LA OFICINA DIOCESANA DE EDUCACIÓN CATÓLICA DE HUÁNUCO
}

\author{
THE VOCATIONAL TRAINING AND THE CHRISTIAN VOCATION OF SERVICE \\ OF TEACHERS OF RELIGIOUS EDUCATION OF THE DIOCESAN OFFICE OF \\ CATHOLIC EDUCATION OF HUÁNUCO
}

CRISPIN Blas C. *

(RECIBIDO 13-07-2013, AcEPTADo 20-09-2013)

\begin{abstract}
RESUMEN
El antecedente del estudio el documento eclesiástico Gravissimum Education es sobre la educación cristiana del Concilio Vaticano II,expresa el derecho de toda persona a una educación integral, esto es, física, moral, intelectual, sexual, social y religiosa. Se reiteran las finalidades y las características de la educación cristiana: la formación humana completa y el progreso en la vida del hombre nuevo en Cristo. Se exponen los derechos y deberes de los padres y la importancia de la familia en la educación, así como los de la sociedad civil y la Iglesia.

El objetivo principal del presente estudio fue determinar el nivel de influencia de la formación profesional cristiana en el desarrollo de la vocación de servicio de los docentes de educación religiosa de la Oficina Diocesana de Educación Católica de Huánuco. Aplicando una encuesta a una muestra de 62 docentes entre hombres y mujeres que laboraban a nivel de la coordinación de la Oficina Diocesana de Educación Católica del distrito de Llata, provincia de Huamalíes, región Huánuco, se llegó a establecer la siguiente conclusión que sí existe una influencia directa y significativa entre la formación profesional y la vocación del servicio de los docentes de educación religiosa de la oficina diocesana de educación católica de Huánuco.
\end{abstract}

Palabras clave: formación profesional cristiana, vocación de servicio, docentes, religión, diócesis, Huánuco

\begin{abstract}
The precedent of the study the ecclesiastic document Gravissimum Education is on the Christian education of the Council Vatican the II, it expresses the right of every person to an integral education, this is, physical, moral, intellectual, sexual, social and religious. There are repeated the purposes and the characteristics of the education it christens: the human complete formation and the progress in the life of the new man in Christ. There are exposed the rights and duties of the parents and the importance of the family in the education, as well as those of the civil society and the Church.
\end{abstract}

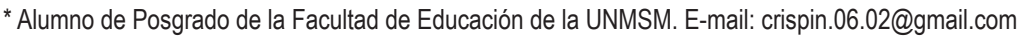


The principal aim of the present study was to determine the level of influence of the Christian vocational training in the development of the vocation of service of the teachers of religious education of the Diocesan Office of Huánuco Catholic Education. Applying a survey to anexample of 62 teachers manand women who were working to level of the coordination of the Diocesan Office Of Catholic Education of Llata district, Huamalíesprovince, region Huánuco, managed to establish the following conclusion that yes exists a direct and significant influence between the vocational training and the vocation of the service of the teachers of religious education of the diocesan office of Huánuco catholic education.

Keyword: christian, vocational training, service vocation, teachers, religion, diocese, Huánuco

\section{INTRODUCCIÓN}

La formación profesional cristiana y la vocación de servicio de los docentes de educación religiosa de la Oficina Diocesana de Educación Católica de Huánuco, tiene como finalidad poner al alcance a todos los actores involucrados en la enseñanza de la educación religiosa, quienes deberán de brindar un servicio profesional de acorde con el avance de la ciencia y la tecnología, capaces de resolver los problemas académicas y morales de los estudiantes de la región.

En muchos centros educativos en los cuales se incluye la Educación Religiosa como parte importante de la actividad académica, se está incurriendo en falta de planeación y atención integral de esta modalidad de educación, lo que impide la garantía de que los docentes sean debidamente capacitados para su labor, la correcta inclusión del tema religioso en el Plan Educativo Institucional y el diseño y adopción de mecanismos y recursos orientados a brindar a los estudiantes una formación religiosa de calidad acorde con el derecho constitucional y legal que le ha sido reconocido.En los diferentes eventos de carácter, social, cultural, religioso, comunal, educativo, deportivo se observa la indiferencia de participación de los docentes de educación religiosa, su misión solo se limita a las actividades del aula; la proyección a la convivencia institucional y a los proyectos de la dinámica social y comunal no es notoria.

Se percibe en la mayoría de los profesionales de la educación y en particular de los profesores de Educación Religiosa la escasa vocación de servicio; como facilitadores, no promueven la participación de los padres de familia en la solución de los problemas educativos y comunales, interviniendo efectivamente en la concientización de los postulados del cristianismo, impartiendo sus conocimientos en forma integral, de carácter científico, cultural, teológico y religioso demostrando su preparación de promotor líder de la sociedad y orientador de los ciudadanos a un futuro provisor. Se ha constatado en la conducta de los docentes de Educación Religiosa la escasa preparación profesional y la carencia de la vocación de servicio, para formar personas inspiradas en la defensa de los principios cristianos en el ejercicio de la profesión y emerger como líder y dirigentes del futuro. La misión limitada del docente afectala evangelización y la efectividad de dar respuestas a los problemas álgidos de la sociedad. 
Por lo expuesto el presente estudio busca determinar:¿Cómo la formación profesional cristiana influye en la vocación de servicio de los docentes de educación religiosa, para formar estudiantes que conozcan y valoren los principios cristianos?

\section{Tipo de investigación}

Por su naturaleza es teórico explicativo.

\section{Hipótesis}

La formación profesional cristiana influye significativamente en el desarrollo de la vocación de servicio de los docentes de Educación Religiosa de la Oficina Diocesana de Educación Católica de Huánuco.

\section{MÉTODOS}

\section{Diseño de la investigación}

Consideramos que la presente investigación sigue un diseño descriptivo No correlacional. Esquematizando matemáticamente tenemos:

$$
\begin{array}{ll}
\mathrm{X}(\text { V.I. }) & =\text { Formación profesional } \\
\mathrm{Y}(\text { V.D. }) & =\text { Vocación de servicio }
\end{array}
$$

Notación funcional: $\quad \mathrm{Y}=\mathrm{f}(\mathrm{X})$

Ello nos indicó la influencia de la formación profesional que existe en la vocación de servicio.

\section{Población y muestra}

La población estuvo constituida por los docentes de educación religiosa que laboran a nivel de la jurisdicción de la diócesis de Huánuco. Fueron aproximadamente 120 docentes entre hombres y mujeres considerados en el padrón de la Oficina Diocesana de Educación Católica.

La muestra está constituida por 62 docentes de educación religiosa, de la coordinación de la Oficina Diocesana de Educación Católica de Huamalíes, seleccionados por el criterio no probabilístico y selección por conveniencia.

\section{Unidad de muestreo y análisis}

La unidad de muestreo y análisis en nuestro estudio es el docente de educación religiosa de la Oficina Diocesana de Educación Católica de Huánuco en estudio.

\section{Técnica de recolección de datos}

Entrevista y el cuestionario elaborados para el propósito de la investigación. 


\section{Medición}

Se empleó para la investigación los ítems politómicos de naturaleza ordinal. Cada uno de estos ítems tiene tres opciones de respuestas, escaladas mediante el procedimiento Likert (deficiente, regular, bueno, excelente).

\section{Técnica de análisis de datos}

Estadísticos descriptivos: frecuencias y porcentajes.

\section{RESULTADOS}

Los resultados a los que arribó la presente investigación se presenta a continuación. Presentaré las respuestas dadas a las preguntas principales de la encuesta y posteriormente haré un análisis e interpretación de las mismas.

\section{FORMACIÓN PROFESIONAL CRISTIANA DIMENSIÓN (1): CAPACIDAD PROFESIONAL - HOMBRES \\ CUADRO N. ${ }^{\circ} 1$}

\begin{tabular}{|c|c|c|c|c|c|}
\hline \multicolumn{6}{|c|}{ 1. ¿Cuál es tu nivel de formación teológica? } \\
\hline & & Frecuencia & Porcentaje & $\begin{array}{l}\text { Porcentaje } \\
\text { válido }\end{array}$ & $\begin{array}{l}\text { Porcentaje } \\
\text { acumulado }\end{array}$ \\
\hline \multirow{3}{*}{ Válidos } & REGULAR & 12 & 40,0 & 40,0 & 40,0 \\
\hline & BUENO & 18 & 60,0 & 60,0 & 100,0 \\
\hline & Total & 30 & 100,0 & 100,0 & \\
\hline
\end{tabular}

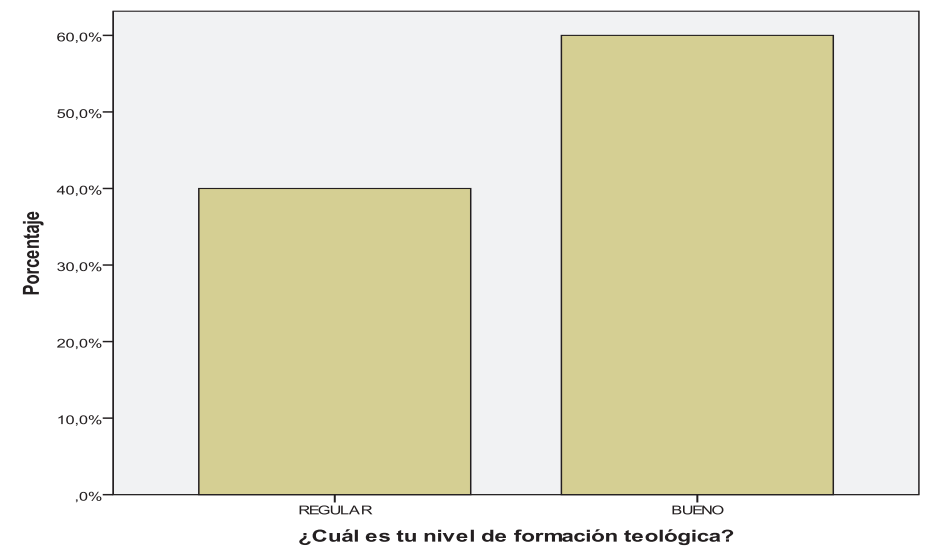

INTERPRETACIÓN: En el gráfico se observa que el $60.00 \%$ de los docentes hombres de educación religiosa cuentan con un nivel de formación teológica buena, el $40.00 \%$ cuentan con un nivel regular. 
CRISPIN BLAS C.

CUADRO N. ${ }^{\circ} 2$

\begin{tabular}{|c|c|c|c|c|c|}
\hline \multicolumn{6}{|c|}{ 2. ¿Cuál es tu nivel de formación filosófica? } \\
\hline & & Frecuencia & Porcentaje & Porcentaje válido & Porcentaje acumulado \\
\hline \multirow[t]{3}{*}{ Válidos } & REGULAR & 13 & 43,3 & 43,3 & 43,3 \\
\hline & BUENO & 17 & 56,7 & 56,7 & 100,0 \\
\hline & Total & 30 & 100,0 & 100,0 & \\
\hline
\end{tabular}

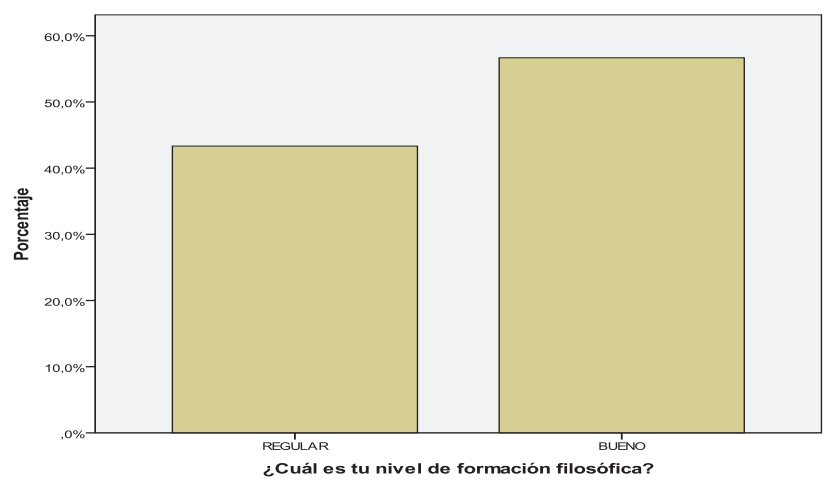

INTERPRETACIÓN: En el gráfico se observa que el 56.7\% de los docentes hombres de educación religiosa cuentan con un nivel de formación filosófica buena, el $43.3 \%$ cuentan con un nivel regular.

\section{CUADRO N. ${ }^{\circ} 3$}

\begin{tabular}{|c|c|c|c|c|c|}
\hline \multicolumn{6}{|c|}{ 3. ¿Cuál es tu nivel del conocimiento de la Doctrina Cristiana? } \\
\hline & & Frecuencia & Porcentaje & Porcentaje válido & Porcentaje acumulado \\
\hline \multirow{4}{*}{ Válidos } & REGULAR & 9 & 30,0 & 30,0 & 30,0 \\
\hline & BUENO & 17 & 56,7 & 56,7 & 86,7 \\
\hline & EXCELENTE & 4 & 13,3 & 13,3 & 100,0 \\
\hline & Total & 30 & 100,0 & 100,0 & \\
\hline
\end{tabular}

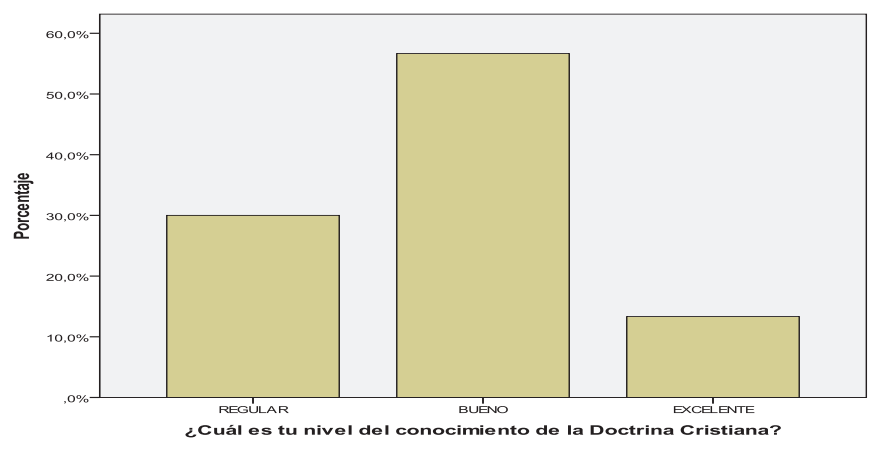


INTERPRETACIÓN: En el gráfico se observa que el 56.7\% de los docentes hombres de educación religiosa cuentan con un nivel del conocimiento de la doctrina cristiana buena, el $30.00 \%$ cuentan con un nivel regular, así mismo el 13.3\% de los docentes cuentan con un nivel de conocimiento de la doctrina cristiana en forma excelente.

\section{CUADRO ${ }^{\circ} 4$}

4. ¿Cuál es tu nivel del conocimiento de los documentos eclesiásticos?

\begin{tabular}{|c|c|c|c|c|c|}
\hline & & Frecuencia & Porcentaje & Porcentaje válido & Porcentaje acumulado \\
\hline \multirow[t]{4}{*}{ Válidos } & REGULAR & 6 & 20,0 & 20,0 & 20,0 \\
\hline & BUENO & 21 & 70,0 & 70,0 & 90,0 \\
\hline & EXCELENTE & 3 & 10,0 & 10,0 & 100,0 \\
\hline & Total & 30 & 100,0 & 100,0 & \\
\hline
\end{tabular}

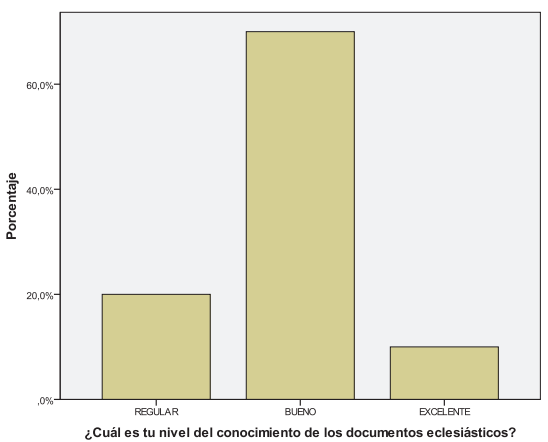

INTERPRETACIÓN: En el gráfico se observa que el $70.0 \%$ de los docentes hombres de educación religiosa cuentan con un nivel del conocimiento de documentos eclesiásticos buena, el $20.00 \%$ cuentan con un nivel regular, así mismo el $10 \%$ de los docentes cuentan con un nivel de conocimiento de los documentos eclesiásticos en forma excelente.

\section{FORMACIÓN PROFESIONAL CRISTIANA \\ DIMENSIÓN (2): FORMACIÓN CRISTIANA - MUJERES \\ CUADRO N. ${ }^{\circ} 5$}

\begin{tabular}{|c|c|c|c|c|c|}
\hline \multicolumn{6}{|c|}{ 1. ¿Cuál es tu nivel de formación cristiana? } \\
\hline & & Frecuencia & Porcentaje & Porcentaje válido & Porcentaje acumulado \\
\hline \multirow[t]{4}{*}{ Válidos } & REGULAR & 6 & 15,8 & 15,8 & 15,8 \\
\hline & BUENO & 26 & 68,4 & 68,4 & 84,2 \\
\hline & EXCELENTE & 6 & 15,8 & 15,8 & 100,0 \\
\hline & Total & 38 & 100,0 & 100,0 & \\
\hline
\end{tabular}




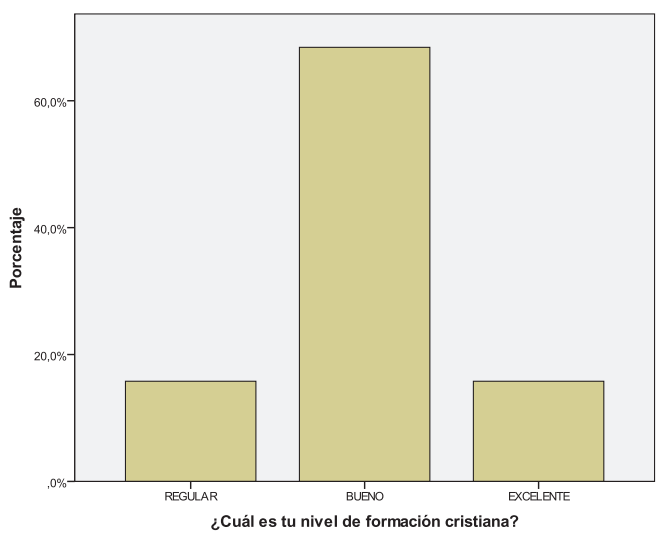

INTERPRETACIÓN: En el gráfico se observa que el $68.4 \%$ de las docentes mujeres de educación religiosa cuentan con un nivel del formación cristiana bueno, el $15.8 \%$ cuentan con un nivel regular y excelente.

CUADRO N. ${ }^{\circ} 6$

\begin{tabular}{|c|c|c|c|c|c|}
\hline \multicolumn{6}{|c|}{ 2. ¿Cuál es tu nivel de la formación integral de la persona? } \\
\hline & & Frecuencia & Porcentaje & Porcentaje válido & Porcentaje acumulado \\
\hline \multirow[t]{4}{*}{ Válidos } & REGULAR & 4 & 10,5 & 10,5 & 10,5 \\
\hline & BUENO & 33 & 86,8 & 86,8 & 97,4 \\
\hline & EXCELENTE & 1 & 2,6 & 2,6 & 100,0 \\
\hline & Total & 38 & 100,0 & 100,0 & \\
\hline
\end{tabular}

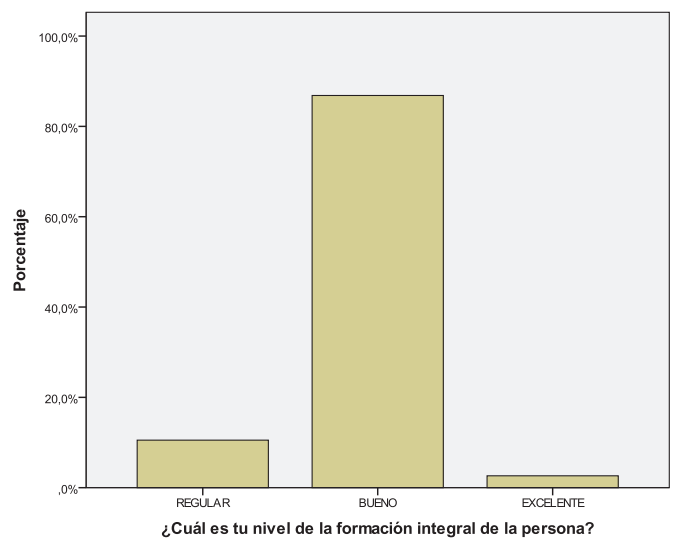

INTERPRETACIÓN: En el gráfico se observa que el $86.8 \%$ de las docentes mujeres de educación religiosa cuentan con un nivel de formación integral de la persona bueno, el $10.5 \%$ cuentan con un nivel regular y el $2.6 \%$ cuenta con un nivel excelente. 
LA FORMACIÓN PROFESIONAL CRISTIANA Y LA VOCACIÓN DE SERVICIO DE LOS DOCENTES DE EDUCACIÓN RELIGIOSA...

\section{CUADRO N. ${ }^{\circ} 7$}

3. ¿Cuál es tu nivel de la formación integral de la persona?

\begin{tabular}{llcccc}
\hline \multirow{4}{*}{ Válidos } & Frecuencia & Porcentaje & Porcentaje válido & Porcentaje acumulado \\
& REGULAR & 4 & 10,5 & 10,5 & 10,5 \\
& BUENO & 33 & 86,8 & 86,8 & 97,4 \\
& EXCELENTE & 1 & 2,6 & 2,6 & 100,0 \\
& Total & 38 & 100,0 & 100,0 & \\
\hline
\end{tabular}

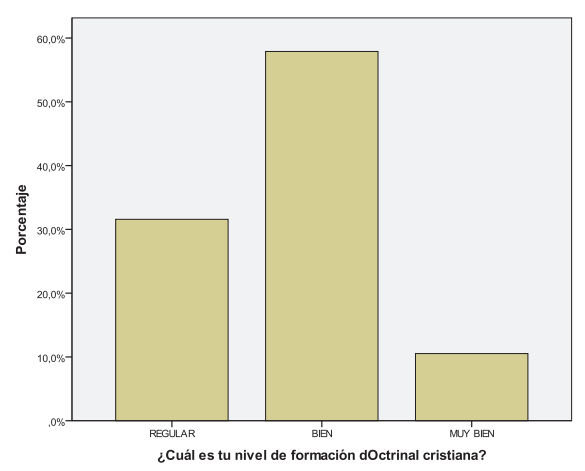

INTERPRETACIÓN: En el gráfico se observa que el 57.9\% de las docentes mujeres de educación religiosa cuentan con un nivel de formación doctrinal cristiana bueno, el $31.6 \%$ cuentan con un nivel regular y el $10.5 \%$ cuenta con un nivel de muy bien.

\section{CUADRO N. ${ }^{\circ} 8$}

\begin{tabular}{lccccc}
\hline \multicolumn{5}{c}{ 4. ¿Cómo fue la educación cristiana que recibió durante su formación profesional? } \\
\hline \multirow{4}{*}{ Válidos } & Frecuencia & Porcentaje & Porcentaje válido & Porcentaje acumulado \\
& DEFICIENTE & 1 & 2,6 & 2,6 & 2,6 \\
& REGULAR & 9 & 23,7 & 23,7 & 26,3 \\
& BIEN & 19 & 50,0 & 50,0 & 76,3 \\
& MUY BIEN & 9 & 23,7 & 23,7 & 100,0 \\
& Total & 38 & 100,0 & 100,0 & \\
\hline
\end{tabular}

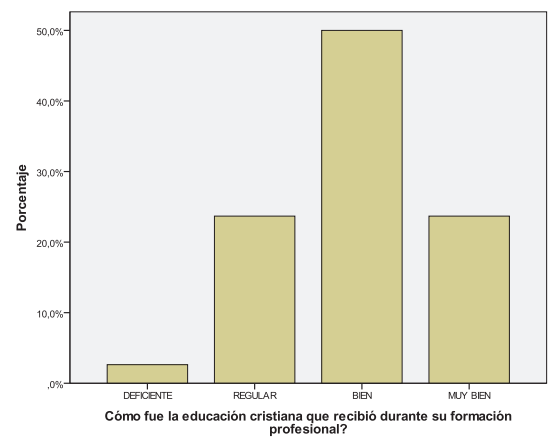


INTERPRETACIÓN: En el gráfico se observa que el 50.0\% de las docentes mujeres de educación religiosa que la educación cristiana que recibió durante su formación profesional fue bien, el $23.7 \%$ han recibido su formación profesional en muy bien y regular asimismo el $2.6 \%$ lo ha recibido de forma deficiente.

\section{CUADRO N. ${ }^{\circ} 9$}

\begin{tabular}{|c|c|c|c|c|c|}
\hline \multicolumn{6}{|c|}{ 5. Se formó a base de principios cristianos. } \\
\hline & & Frecuencia & Porcentaje & Porcentaje válido & Porcentaje acumulado \\
\hline \multirow[t]{4}{*}{ Válidos } & REGULAR & 8 & 21,1 & 21,1 & 21,1 \\
\hline & BIEN & 21 & 55,3 & 55,3 & 76,3 \\
\hline & MUY BIEN & 9 & 23,7 & 23,7 & 100,0 \\
\hline & Total & 38 & 100,0 & 100,0 & \\
\hline
\end{tabular}

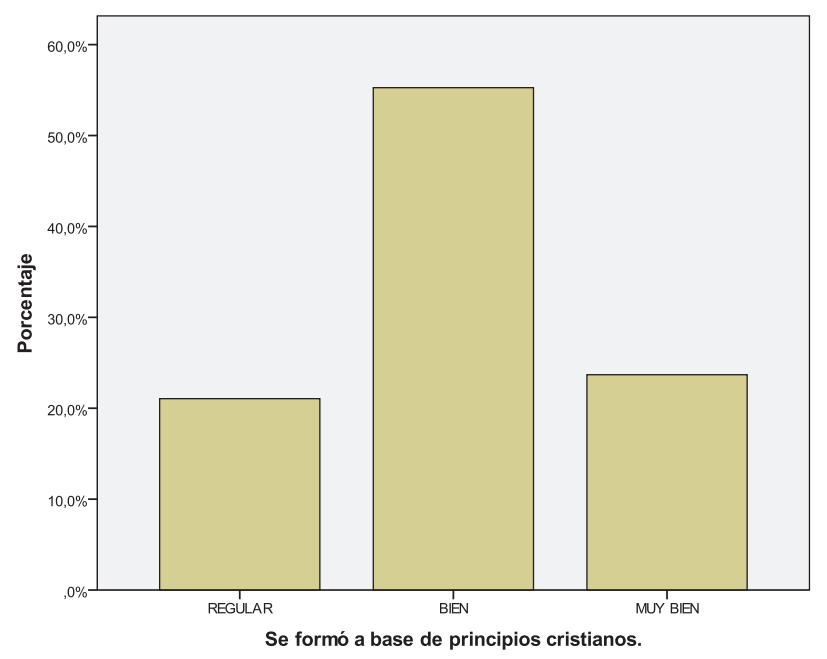

INTERPRETACIÓN: En el gráfico se observa que el $55.3 \%$ de las docentes mujeres de educación religiosa se formó bien a base de principios cristianos, el $23.7 \%$ se ha formado muy bien $21.1 \%$ se formó de manera regular.

$$
\text { CUADRO N. }{ }^{\circ} 10
$$

\begin{tabular}{lccccc}
\hline \multicolumn{5}{c}{ 6. ¿Tuvo una formación acorde al avance de la ciencia y tecnología? } \\
\hline \multirow{4}{*}{ Válidos } & Frecuencia & Porcentaje & Porcentaje válido & Porcentaje acumulado \\
& DEFICIENTE & 5 & 13,2 & 13,2 & 13,2 \\
& REGULAR & 13 & 34,2 & 34,2 & 47,4 \\
& BIEN & 19 & 50,0 & 50,0 & 97,4 \\
& MUY BIEN & 1 & 2,6 & 2,6 & 100,0 \\
& Total & 38 & 100,0 & 100,0 & \\
\hline
\end{tabular}




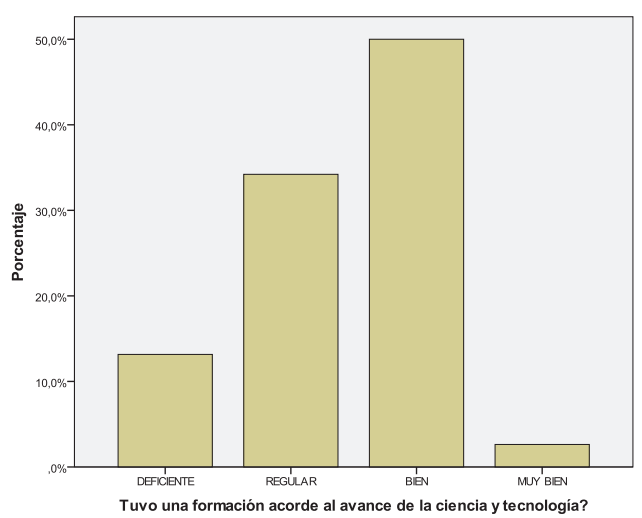

INTERPRETACIÓN: En el gráfico se observa que el 50.0\% de las docentes mujeres de educación religiosa tuvo su formación bien de acorde al avance de la ciencia y tecnología el $34.2 \%$ tuvo su formación de una forma regular, el 13.2\% se formó de manera deficiente y el $2.6 \%$ tuvo muy bien una formación de acorde al avance la ciencia y tecnología.

CUADRO N. ${ }^{\circ} 11$

\begin{tabular}{lccccc}
\hline \multicolumn{6}{c}{ 7. ¿Practicas la fe cristiana? } \\
\hline \multirow{4}{*}{ Válidos } & Frecuencia & Porcentaje & Porcentaje válido & Porcentaje acumulado \\
& Sí & 29 & 76,3 & 76,3 & 76,3 \\
& A VECES & 9 & 23,7 & 23,7 & 100,0 \\
& Total & 38 & 100,0 & 100,0 & \\
\hline
\end{tabular}

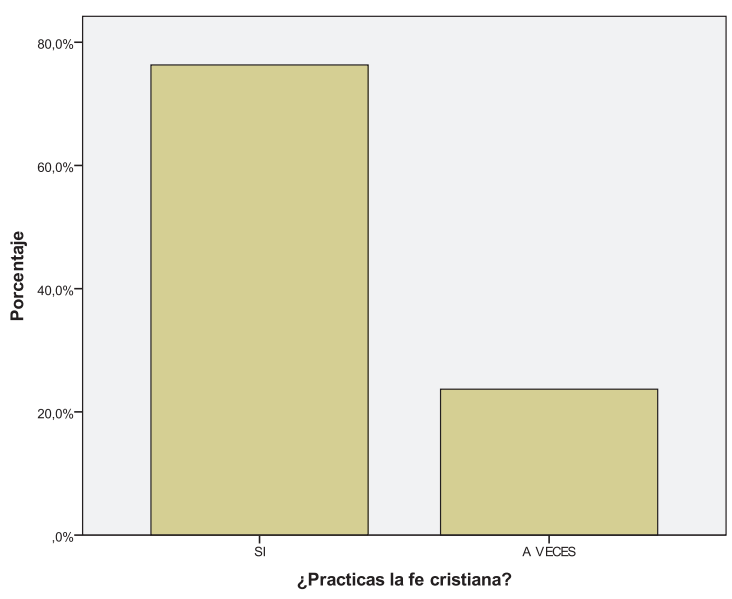

INTERPRETACIÓN: En el gráfico se observa que el 76.3\% de docentes mujeres de educación religiosa sí practica la fe cristiana y el $23.7 \%$ a veces práctica la fe cristiana. 


\section{VARIABLE DEPENDIENTE: VOCACIÓN DE SERVICIO DIMENSIÓN (1): COMPROMISO CRISTIANO - HOMBRES}

\section{CUADRO N. ${ }^{\circ} 12$}

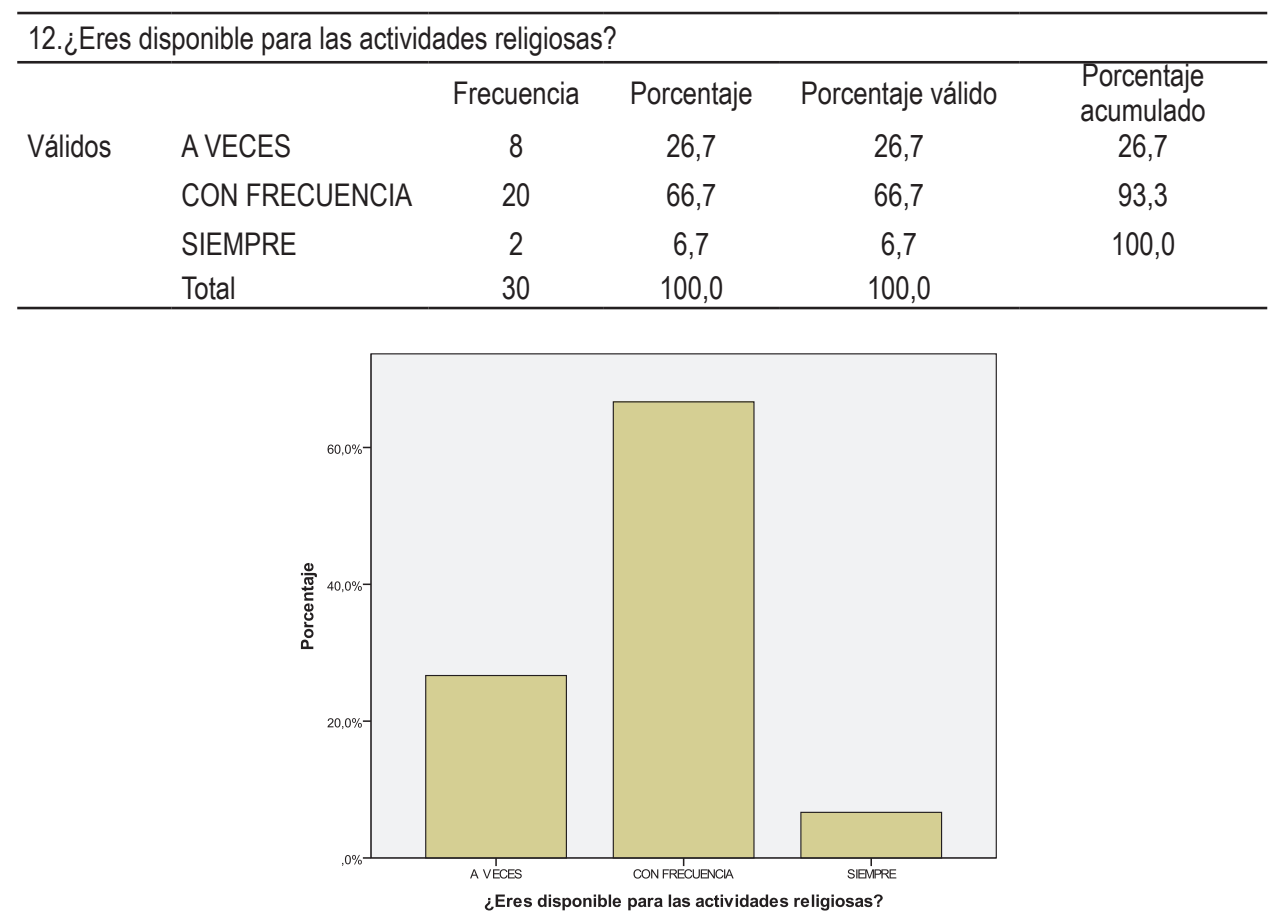

INTERPRETACIÓN: En el gráfico se observa que el $66.7 \%$ de los docentes hombres de educación religiosa con frecuencia se encuentran disponibles para las actividades religiosas, el $26.7 \%$ a veces se encuentra disponible para las actividades religiosas, así mismo el $6.7 \%$ de los docentes siempre se encuentran disponibles.

\section{CUADRO N. 13}

\begin{tabular}{|c|c|c|c|c|c|}
\hline \multicolumn{6}{|c|}{ 13. ¿Realizas la pastoral educativa en tu I.E. o en la parroquia? } \\
\hline & & Frecuencia & Porcentaje & Porcentaje válido & Porcentaje acumulado \\
\hline \multirow[t]{5}{*}{ Válidos } & NUNCA & 1 & 3,3 & 3,3 & 3,3 \\
\hline & A VECES & 8 & 26,7 & 26,7 & 30,0 \\
\hline & CON FRECUENCIA & 18 & 60,0 & 60,0 & 90,0 \\
\hline & SIEMPRE & 3 & 10,0 & 10,0 & 100,0 \\
\hline & Total & 30 & 100,0 & 100,0 & \\
\hline
\end{tabular}




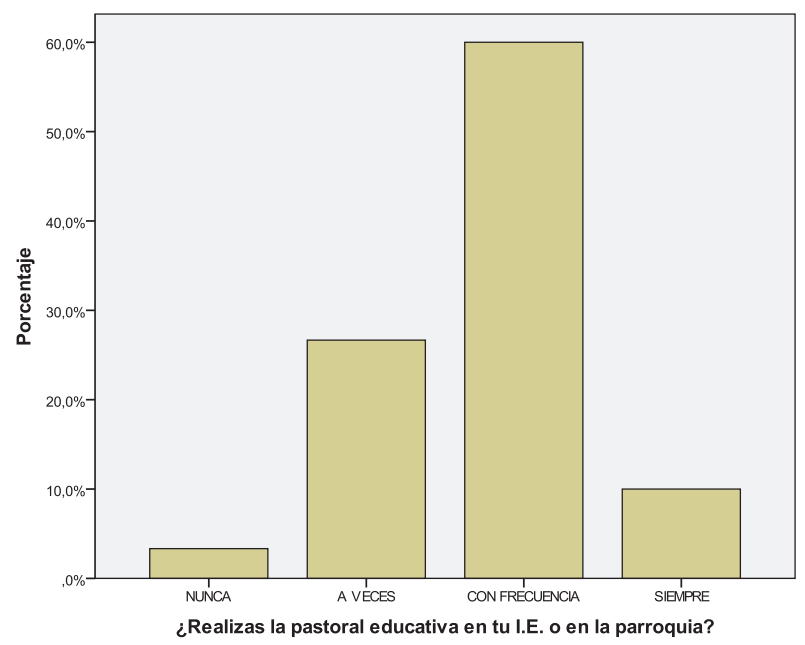

INTERPRETACIÓN: En el gráfico se observa que el $60.0 \%$ de los docentes hombres de educación religiosa con frecuencia se realizan la pastoral educativa en su Institución educativa o en la parroquia, el $26.7 \%$ a veces realizan la pastoral, el $10.0 \%$ a veces lo realiza, así mismo el $3.3 \%$ de los docentes nunca lo realizan.

\section{CUADRO $\mathrm{N}^{\circ} 14$}

\begin{tabular}{llcccc}
\hline \multicolumn{6}{l}{ 14.¿Te identificas con el área de Educación Religiosa? } \\
\hline \multirow{6}{*}{ Válidos } & Frecuencia & Porcentaje & Porcentaje válido & Porcentaje acumulado \\
& NUNCA & 1 & 3,3 & 3,3 & 3,3 \\
& A VECES & 12 & 40,0 & 40,0 & 43,3 \\
& CON FRECUENCIA & 13 & 43,3 & 43,3 & 86,7 \\
& SIEMPRE & 4 & 13,3 & 13,3 & 100,0 \\
& Total & 30 & 100,0 & 100,0 & \\
\hline
\end{tabular}

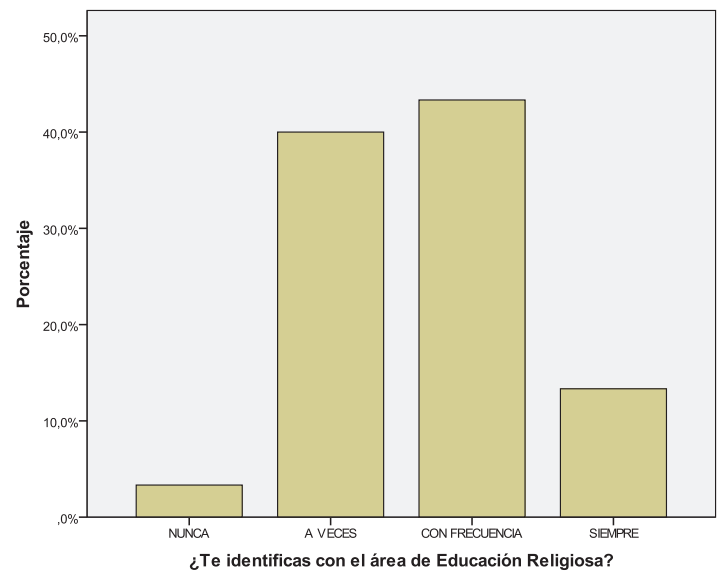


INTERPRETACIÓN: En el gráfico se observa que el 43.3\% de los docentes hombres de educación religiosa con frecuencia se identifican con el área de educación religiosa, el $40.0 \%$ a veces se identifican, el $13.3 \%$ siempre se identifican, así mismo el $3.3 \%$ de los docentes responden que nunca se identifican.

\section{CUADRO N. ${ }^{\circ} 15$}

\begin{tabular}{|c|c|c|c|c|c|}
\hline \multicolumn{6}{|c|}{ 15.¿Te identificas con la I.E. y con la parroquia donde laboras? } \\
\hline & & Frecuencia & Porcentaje & Porcentaje válido & Porcentaje acumulado \\
\hline \multirow[t]{5}{*}{ Válidos } & NUNCA & 1 & 3,3 & 3,3 & 3,3 \\
\hline & A VECES & 9 & 30,0 & 30,0 & 33,3 \\
\hline & CON FRECUENCIA & 13 & 43,3 & 43,3 & 76,7 \\
\hline & SIEMPRE & 7 & 23,3 & 23,3 & 100,0 \\
\hline & Total & 30 & 100,0 & 100,0 & \\
\hline
\end{tabular}

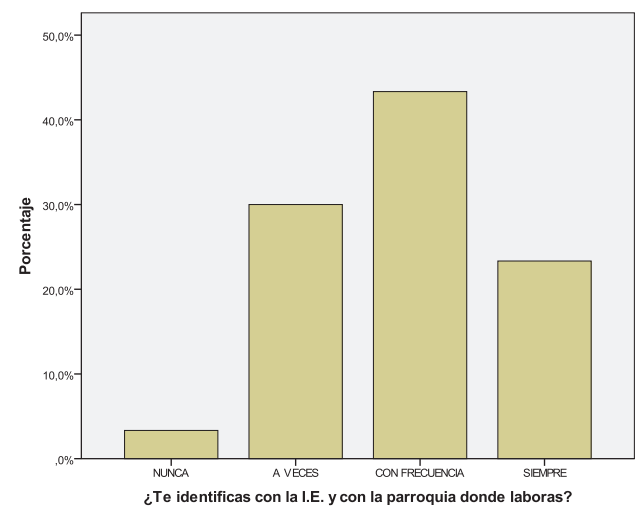

INTERPRETACIÓN: En el gráfico se observa que el $43.3 \%$ de los docentes hombres de educación religiosa con frecuencia se identifican con la institución educativa y con la parroquia donde labora, el $23.3 \%$ a veces se identifican con la institución educativa y con la parroquia donde labora, el $23.3 \%$ de los docentes siempre se identifican con ambas instituciones y el $3.3 \%$ nunca se identifican ni con la institución educativa y ni con la parroquia donde laboran.

\section{CUADRO N. ${ }^{\circ} 16$}

\begin{tabular}{llcccc}
\hline 16 ¿Me siento comprometido a trabajar con vocación de servicio? & & \\
\hline \multirow{4}{*}{ Válidos } & Frecuencia & Porcentaje & Porcentaje válido & Porcentaje acumulado \\
& A VECES & 11 & 36,7 & 36,7 & 36,7 \\
& CON FRECUENCIA & 14 & 46,7 & 46,7 & 83,3 \\
& SIEMPRE & 5 & 16,7 & 16,7 & 100,0 \\
& Total & 30 & 100,0 & 100,0 & \\
\hline
\end{tabular}




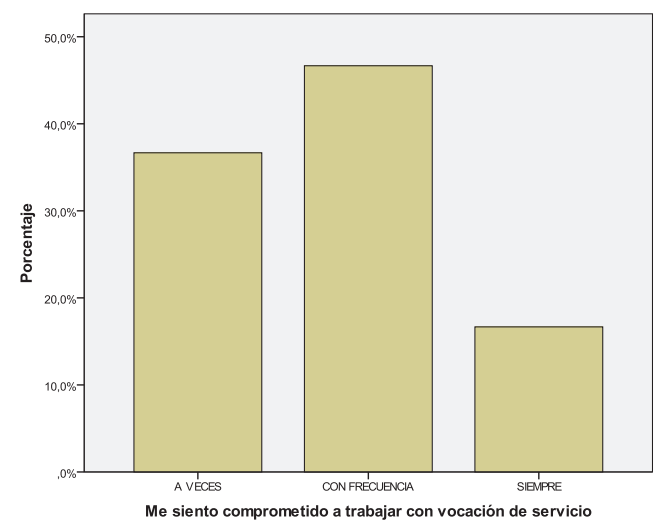

INTERPRETACIÓN: En el gráfico se observa que el $46.7 \%$ de los docentes hombres de educación religiosa con frecuencia se sienten comprometidos a trabajar con vocación de servicio, el $36.7 \%$ a veces se sienten comprometido a trabajar con vocación de servicio y el $16.7 \%$ de los docentes siempre se sienten comprometidos en trabajar con vocación de servicio.

\section{VARIABLE DEPENDIENTE: VOCACIÓN DE SERVICIO}

\section{DIMENSIÓN (2): TESTIMONIO CRISTIANO-MUJERES}

\section{CUADRO N. ${ }^{\circ} 17$}

\begin{tabular}{lccccc}
\hline 1. ¿Eres coherente con lo que dices y haces? & & & \\
\hline \multirow{4}{*}{ Válidos } & Frecuencia & Porcentaje & Porcentaje válido & Porcentaje acumulado \\
& A VECES & 5 & 13,2 & 13,2 & 13,2 \\
& CON FRECUENCIA & 28 & 73,7 & 73,7 & 86,8 \\
& SIEMPRE & 5 & 13,2 & 13,2 & 100,0 \\
& Total & 38 & 100,0 & 100,0 & \\
\hline
\end{tabular}

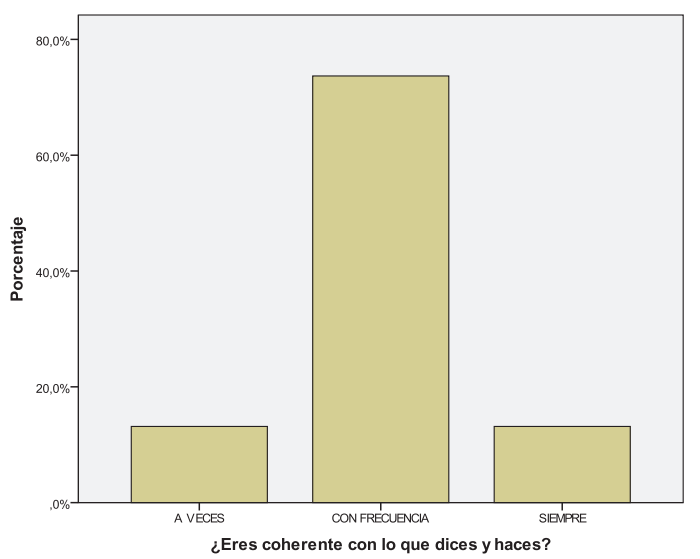


INTERPRETACIÓN: En el gráfico se observa que el $73.7 \%$ de las docentes mujeres de educación religiosa con frecuencia son coherentes con lo que dicen y hacen, el $13.2 \%$ siempre y a veces son coherentes.

\section{CUADRO N. ${ }^{\circ} 18$}

\begin{tabular}{llcccc}
\hline 1. ¿Demuestras la coherencia de vida cristiana? & & & \\
\hline \multirow{4}{*}{ Válidos } & Frecuencia & Porcentaje & Porcentaje válido & Porcentaje acumulado \\
& A VECES & 5 & 13,2 & 13,2 & 13,2 \\
& CON FRECUENCIA & 23 & 60,5 & 60,5 & 73,7 \\
& SIEMPRE & 10 & 26,3 & 26,3 & 100,0 \\
& Total & 38 & 100,0 & 100,0 & \\
\hline
\end{tabular}

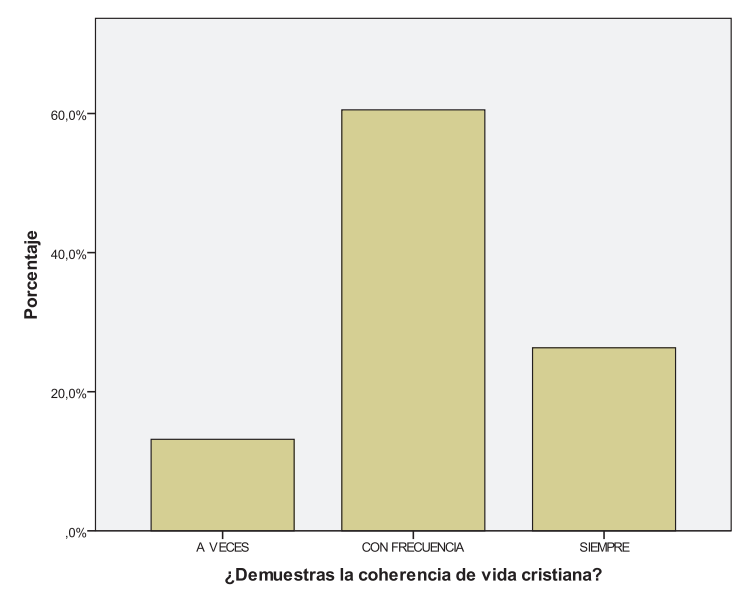

INTERPRETACIÓN: En el gráfico se observa que el $60.5 \%$ de las docentes mujeres de educación religiosa con frecuencia demuestran la coherencia de vida cristiana, el $26.3 \%$ siempre demuestran coherencia y el $13.2 \%$ a veces demuestran coherencia.

\section{CUADRO $\mathrm{N}^{\circ} 19$}

\begin{tabular}{llcccc}
\hline 2. ¿Eres empático con tus estudiantes? & & & & \\
\hline \multirow{4}{*}{ Válidos } & Frecuencia & Porcentaje & Porcentaje válido & Porcentaje acumulado \\
& A VECES & 9 & 23,7 & 23,7 & 23,7 \\
& CON FRECUENCIA & 12 & 31,6 & 31,6 & 55,3 \\
& SIEMPRE & 17 & 44,7 & 44,7 & 100,0 \\
& Total & 38 & 100,0 & 100,0 & \\
\hline
\end{tabular}


LA FORMACIÓN PROFESIONAL CRISTIANA Y LA VOCACIÓN DE SERVICIO DE LOS DOCENTES DE EDUCACIÓN RELIGIOSA...

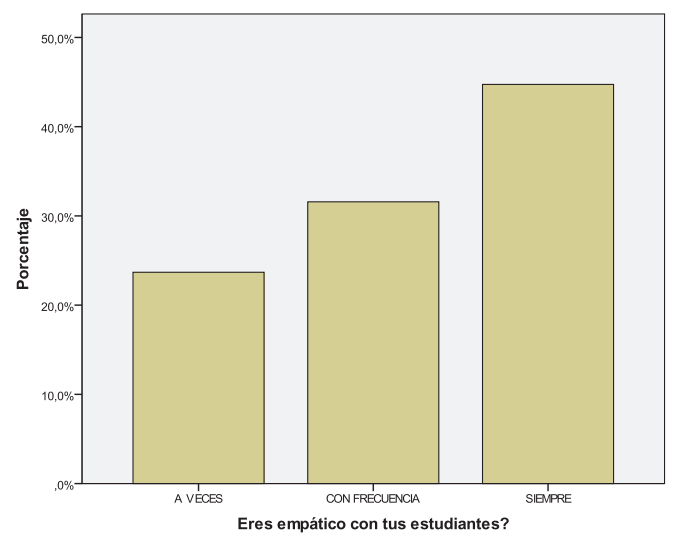

INTERPRETACIÓN: En el gráfico se observa que el $44.7 \%$ de las docentes mujeres de educación religiosa siempre son empáticas con sus estudiantes, el 31.6\% con frecuencia son empáticos y el $23.7 \%$ a veces son empáticos.

\section{CUADRO N. ${ }^{\circ} 20$}

\begin{tabular}{|c|c|c|c|c|c|}
\hline \multicolumn{6}{|c|}{ 4. ¿Promueves la empatía social? } \\
\hline & & Frecuencia & Porcentaje & Porcentaje válido & Porcentaje acumulado \\
\hline \multirow[t]{4}{*}{ Válidos } & A VECES & 9 & 23,7 & 23,7 & 23,7 \\
\hline & CON FRECUENCIA & 16 & 42,1 & 42,1 & 65,8 \\
\hline & SIEMPRE & 13 & 34,2 & 34,2 & 100,0 \\
\hline & Total & 38 & 100,0 & 100,0 & \\
\hline
\end{tabular}

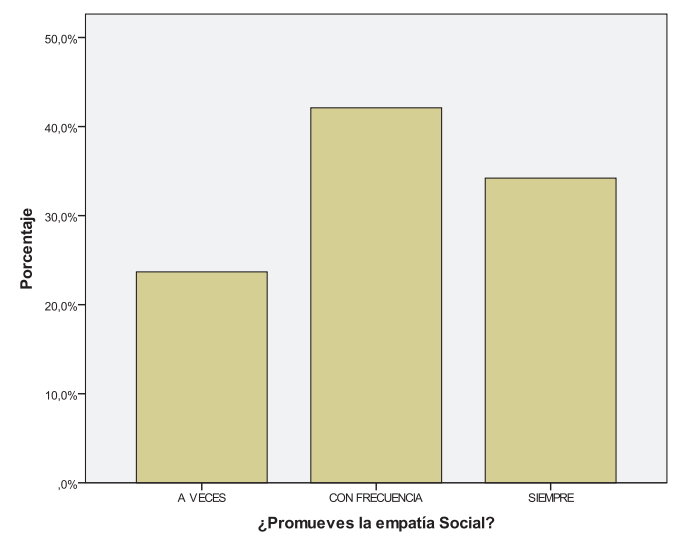

INTERPRETACIÓN: En el gráfico se observa que el $42.1 \%$ de las docentes mujeres de educación religiosa con frecuencia promueven la empatía social, el $34.2 \%$ siempre promueven la empatía y el $23.7 \%$ a veces promueven la empatía. 


\section{CONCLUSIONES}

1) La Formación profesional influye significativamente con la vocación del servicio de los docentes de Educación Religiosa de la Oficina Diocesana de Educación Católica de Huánuco, con lo que cumple con la hipótesis planteada en la investigación al 72.4\% de confianza.

2) La capacidad profesional influye directa y significativamente con la vocación de servicio, con lo que cumple con la hipótesis planteada en la investigación al 77.7\% de confianza.

3) La formación cristiana influye directa y significativamente con la vocación de servicio, con lo que cumple con la hipótesis planteada en la investigación al 75.3\% de confianza.

\section{REFERENCIAS BIBLIOGRÁFICAS}

\section{Bibliografía de la investigación}

ÁVILA ACOSTA, R. B. (2001). Metodología de la Investigación. Cómo elaborar la Tesis y/o investigación. Estudios y Ediciones R. A. Lima-Perú.

ARNAL AGUSTÍN, Justo (2000). Perspectivas contemporáneas en Metodología de la Investigación. Cuadernos Pedagógicos. Lima-Perú.

BARRIGA HERNÁNDEZ, Carlos (1993). Elementos de Investigación científica. Editorial Aula Nueva. Lima-Perú.

\section{Bibliografía del tema}

ÁLVAREZ, J.(2001). El análisis de un modelo de educación integral. Tesis Doctoral. España: Editorial Universidad de Granada.

BOLÍVAR, A. (2005). El lugar de la ética profesional en la formación universitaria. Revista Mexicana de Investigación Educativa.

BOMBINO, L. (2004). Conceptos fundamentales en ética, valores, axiología y educación. En Bombino (coord.). El saber ético de ayer a hoy. Tomo II (pp. 47-71). La Habana: Editorial Félix Varela.

\section{Artículo}

Chávez Zamora, José. Creación de zonas de desarrollo del metapensamiento con la escritura productiva o creativa.

\section{Revista}

Revista de Investigación en Educación y Psicología. 